\title{
ON A PROBLEM OF DOOB ABOUT THE FINE TOPOLOGY AND NORMAL FUNCTIONS
}

\author{
J. S. HWANG ${ }^{1}$
}

\begin{abstract}
Early in the sixties, Joseph L. Doob proved that if $f(z)$ is a normal function in a disk, then every angular cluster value at a boundary point is also a fine cluster value at that point. He then asked whether or not the converse of this theorem is true.

It was pointed out by the referee that Choquet was the first one to show that the converse of the above theorem is false. His example was published by Brelot and Doob (see [2, p. 404]).

Recently, the referee suggests the question as to whether a bounded analytic function can actually have an angular limit but not a fine limit. We construct a Blaschke product which has the fine cluster value 0 and the angular limit 1 at the same boundary point. This answers both questions of Doob and the referee.
\end{abstract}

1. Introduction. Let $H=\{z: \operatorname{Re} z>0\}$ be the right half-plane and let $\partial H$ be the boundary of $H$. Then the Martin boundary is simply the union $\partial H \cup\{\infty\}$. As usual (see Brelot and Doob [2, p. 397]), a set $S \subset H$ is minimally thin at a point $p \in \partial H \cup\{\infty\}$ if it satisfies

$$
R_{k_{p}}^{S}(q) \not k_{p}(q)
$$

where $k_{p}$ is a minimal harmonic function with pole at $p$ and the left-hand side is the reduced function of $k_{p}$ on $S$ with respect to $H$. Since the functions on both sides of (1) are conformally invariant, so is the minimal thinness. For this reason, instead of solving Doob's problem [4, p. 529] in a disk, we shall do it in a half-plane and we shall concentrate on the point at $\infty$. In this case, (1) becomes

$$
R_{x}^{S}(z) \neq x \text {, where } z=x+i y \text { and } x>0 .
$$

Notice that if a set is thin at $\infty$ in the ordinary sense, then it is minimally thin there due to a theorem of Jackson [7, Theorem 4]. Therefore, to solve Doob's problem, we need only consider the minimal thinness. This is the reason why we do not introduce the notion of ordinary thinness in the sense of Cartan and Brelot; see for instance [7, p. 203].

As usual (see Brelot and Doob [2, p. 401]), a function $f(z)$ has a fine cluster value $v$ at $\infty$ if there is a set $S \subset H$ which is not minimally thin at $\infty$ and satisfies $\lim _{z \rightarrow \infty} f(z)=v$, where $z \in S$.

Received by the editors February 25, 1980 and, in revised form, June 26, 1980.

AMS (MOS) subject classifications (1970). Primary 31A15; Secondary 30A74.

Key words and phrases. Fine topology, normal function, Blaschke product, cluster value.

${ }^{1}$ I am indebted to the referee for his pointing out a mistake in my manuscript and many valuable comments in this research. 
Moreover, we say that a function $f$ has an angular cluster value $v$ at $\infty$ if there is a Stolz angle of the form

$$
\Delta_{e}=\{z:|\arg z|<\pi / 2-\varepsilon, 0<a<|z|<\infty\}, \text { where } \varepsilon>0,
$$

and a sequence of points $p_{n} \in \Delta_{e}$ such that $\lim _{n \rightarrow \infty} p_{n}=\infty$ and $\lim _{n \rightarrow \infty} f\left(p_{n}\right)=v$.

Since a bounded holomorphic function is normal in the sense of Lehto and Virtanen [8], the problem of Doob will be solved by the following.

THEOREM. Let $B\left(z,\left\{z_{n}\right\}\right)$ be the Blaschke product defined by

$$
B\left(z,\left\{z_{n}\right\}\right)=\prod_{n=1}^{\infty} \frac{\left(z_{n}-z\right)\left(\bar{z}_{n}-z\right)}{\left(\bar{z}_{n}+z\right)\left(z_{n}+z\right)},
$$

where $z_{n}=2^{n}\left(n^{-1 / 2}+i\right), n=1,2, \ldots$ Then $B$ has the fine cluster value 0 and the angular limit 1 on the same point at $\infty$.

2. Minimal thinness. Following Lelong-Ferrand [9], we let $\lambda_{s}$ be the fundamental distribution of a bounded set $S \subset H$ such that the Green potential

$$
\hat{R}_{x}^{S}(z)=\int_{\partial S} \log \left|\frac{w+\bar{z}}{w-z}\right| d \lambda_{S}(w)=x, \quad \text { q.e. for } z=x+i y \in S,
$$

and define the Green energy of $\lambda_{s}$ to be

$$
\gamma(S)=\int_{\partial S} \hat{R}_{x}^{S}(z) d \lambda_{S}(z)
$$

We say that a set $E \subset H$ is minimally thin at $\infty$ iff

$$
\sum_{n=1}^{\infty} \gamma\left(E_{n}\right) t^{-2 n}<\infty, \text { for some } t>1 \text {, }
$$

where $E_{n}=E \cap\left\{z: t^{n}<|z|<t^{n+1}\right\}$.

Notice that the above two definitions (2) and (6) are equivalent; see Brelot $[3, \mathrm{p}$. 152].

We now consider a disk $D_{r}(z)$ of radius $r$ with center at $z$. Clearly, if $w \in \partial D_{r}(z)$ and $x>2 r$, where $x=\operatorname{Re} z$, then we have

$$
\left(\frac{2 x}{r}\right)^{1 / 2}<\frac{2 x-r}{r}<\left|\frac{w+\bar{z}}{w-z}\right|<\frac{2 x+r}{r}<\left(\frac{2 x}{r}\right)^{2} \text {. }
$$

It follows from (4) that

$$
\frac{1}{2} \lambda(D) \log \frac{2 x}{r}<x<2 \lambda(D) \log \frac{2 x}{r},
$$

where $D=D_{r}(z)$ and $\lambda=\lambda_{D}$.

Moreover, from (5), we get

$$
(x / 2) \lambda(D)<(x-r) \lambda(D)<\gamma(D)<(x+r) \lambda(D)<2 x \lambda(D) .
$$

Due to (7) and (8), we find that

$$
\frac{x^{2}}{4}\left(\log \frac{2 x}{r}\right)^{-1}<\gamma(D)<4 x^{2}\left(\log \frac{2 x}{r}\right)^{-1} .
$$

Combining (6) and (9), we obtain the following result of Essén and Jackson [5, Theorem 3.2]. 
LEMMA 1. Let $D_{n}=\left\{z:\left|z-z_{n}\right|<r_{n}, t^{n}<|z|<t^{n+1}\right\}$, where $t>1$ is fixed and $\operatorname{Re} z_{n}=x_{n}>2 r_{n}, n=1,2, \ldots$ Then the union $D=\cup D_{n}$ is minimally thin at $\infty$ iff

$$
\sum_{n=1}^{\infty} \frac{x_{n}^{2}}{\left|z_{n}\right|^{2} \log \left(2 x_{n} / r_{n}\right)}<\infty .
$$

3. Hyperbolic metric. As usual (see Hille [6, p. 241]), the hyperbolic metric between two points $z, w \in H$ is defined by

$$
d(z, w)=\frac{1}{2} \log \frac{|\bar{z}+w|+|z-w|}{|\bar{z}+w|-|z-w|} .
$$

Since hyperbolic metric is conformally invariant and every bounded function is normal, by a result of Bagemihl and Seidel [1, Lemma 1], we obtain immediately the following.

LEMMA 2. If $f(z)$ is a bounded holomorphic function in $H$ and if $\left\{z_{n}\right\}$ and $\left\{w_{n}\right\}$ are two sequences of points in $H$ for which $d\left(z_{n}, w_{n}\right) \rightarrow 0$, as $n \rightarrow \infty$, then the existence of one limit, say, $\lim _{n \rightarrow \infty} f\left(z_{n}\right)=c$, implies the other $\lim _{n \rightarrow \infty} f\left(w_{n}\right)=c$.

4. Proof of the theorem. With the help of the above two lemmas, we are now able to prove our theorem. In view of (3), those zeros $z_{n}$ of the Blaschke product $B$ satisfies

$$
\sum_{n=1}^{\infty} \frac{\operatorname{Re} z_{n}}{1+\left|z_{n}\right|^{2}}<\infty,
$$

and therefore $B$ is well defined; see Hille [6, p. 457].

We shall prove that the product $B$ has the fine cluster value 0 at $\infty$. To see this, we write $x_{n}=2^{n} n^{-1 / 2}, y_{n}=2^{n}$, and $r_{n}=2^{n} n^{-2}$, and we denote $D_{n}$ the disk of radius $r_{n}$ with center at $z_{n}$. It follows from (10) that

$$
\sum_{n=1}^{\infty} \frac{x_{n}^{2}}{\left|z_{n}\right|^{2} \log \left(2 x_{n} / r_{n}\right)}=\sum_{n=1}^{\infty} \frac{1}{(1+n) \log \left(2 n^{3 / 2}\right)}=\infty \text {. }
$$

This shows that the union $D=\cup D_{n}$ is not minimally thin at $\infty$ due to Lemma 1 .

It remains to show that

$$
\lim _{z \rightarrow \infty} B(z)=0, \quad \text { where } z \in D \text { and } B(z)=B\left(z,\left\{z_{n}\right\}\right) .
$$

For this, we let $w_{n}=z_{n}+r_{n} e^{i \theta}$; then we have

$$
\left|\left(w_{n}-z_{n}\right) /\left(\bar{w}_{n}+z_{n}\right)\right|<r_{n} /\left(2 x_{n}-r_{n}\right)=1 /\left(2 n^{3 / 2}-1\right) .
$$

In view of (11), we find that $d\left(z_{n}, w_{n}\right) \rightarrow 0$ as $n \rightarrow \infty$. According to Lemma 2, we conclude the assertion (12) and therefore $B$ has the fine cluster value 0 at $\infty$.

Finally, we shall prove that the product $B$ has the angular limit 1 at $\infty$. According to a theorem of Lehto and Virtanen [8, Theorem 2], it is sufficient to prove that

$$
\lim _{x \rightarrow \infty} B(x)=1, \quad \text { where } x>0
$$


By a simple computation, the product $B$ defined in (3) can be written as

$$
B(x)=\prod_{n=1}^{\infty}\left(1-f_{n}(x)\right), \quad f_{n}(x)=\frac{2^{n+2} n^{-1 / 2} x}{\left(2^{n} n^{-1 / 2}+x\right)^{2}+2^{2 n}}
$$

Clearly, each function $f_{n}(x)$ satisfies

$$
\begin{gathered}
f_{n}(x)<\frac{2^{n+2} n^{-1 / 2} x}{x^{2}+2^{2 n}}<2 n^{-1 / 2}, \text { for all } x>0, \\
\lim _{x \rightarrow \infty} f_{n}(x)=0, \quad n=1,2, \ldots
\end{gathered}
$$

We shall need the following basic inequality

$$
e^{-2 y}<1-y<e^{-y}, \text { for } 0<y<\frac{1}{2} \text {. }
$$

It follows from (14), (15), (16), and (17) that there is a positive number $P$ such that $x>P$ implies

$$
e^{-2 \sigma(x)}<B(x)<e^{-\sigma(x)}, \quad \sigma(x)=\sum_{n=1}^{\infty} f_{n}(x) .
$$

From (16) and (18), we can see that the assertion (13) will be proved if we can show that the series $\sigma(x)$ converges to 0 . For this, we let $\sigma(x)=\sigma_{1}(x)+\sigma_{2}(x)=$ $\sum_{n=1}^{k} f_{n}(x)+\sum_{n=k+1}^{\infty} f_{n}(x)$. Then by (15), we have

$$
\begin{aligned}
\sigma_{2}(x) & <\sum_{n=k+1}^{\infty} \frac{4 x 2^{n}}{\left(x^{2}+2^{2 n}\right) \sqrt{n}}<\frac{8 x}{\sqrt{k}} \int_{k}^{\infty} \frac{2^{y} d y}{x^{2}+\left(2^{y}\right)^{2}} \\
& <\frac{8 x}{\sqrt{k}} \int_{1}^{\infty} \frac{d u}{x^{2}+u^{2}}<4 \pi / \sqrt{k} .
\end{aligned}
$$

For any $\varepsilon>0$, we first choose $k$ so that $4 \pi / \sqrt{k}<\varepsilon / 2$ and then by (16), we choose $\delta>0$ so that $\sigma_{1}(x)<\varepsilon / 2$ if $x>\delta$. Therefore we obtain $\sigma(x)<\varepsilon$ if $x>\delta$ which means that $\lim _{x \rightarrow \infty} \sigma(x)=0$. This yields (13) and the proof is complete.

\section{REFERENCES}

1. F. Bagemihl and W. Seidel, Sequential and continuous limits of meromorphic functions, Ann. Acad. Sci. Fenn. Ser. AI 280 (1960),

2. M. Brelot and J. L. Doob, Limites angulaires et limites fines, Ann. Inst. Fourier Grenoble 13 (1963), 395-415.

3. M. Brelot, On topologies and boundaries in potential theory, Lecture Notes in Math., vol. 175, Springer-Verlag, Berlin and New York, 1971.

4. J. L. Doob, Conformally invariant cluster value theory, Illinois J. Math. 5 (1961), 521-549.

5. M. Essen and H. L. Jackson, On the covering properties of certain exceptional sets in a half space, Hiroshima J. Math. 10 (1980), 233-262.

6. E. Hille, Analytic function theory. II, Ginn, Boston, Mass., 1962.

7. H. L. Jackson, Some results on thin sets in a half plane, Ann. Inst. Fourier Grenoble 20 (1970), 201-218.

8. O. Lehto and K. I. Virtanen, Boundary behaviour and normal meromorphic functions, Acta Math. 97 (1957), 47-65.

9. J. Lelong-Ferrand, Etude au voisinage de la frontière des fonctions surharmoniques pasitives dans un demi-espace, Ann. École Norm. Sup. 66 (1949), 125-159.

Instrtute of Mathematics, Academa Sinica, Taipei, Tarwan, Republic of China 